\title{
Detection of Tumours Using Breast Surface Thermal Patterns
}

\author{
Y.S. Yu ${ }^{1}$, G.S. Hu ${ }^{1}$, S.C. Fok ${ }^{1}$ \\ ${ }^{1}$ Sichuan University Pittsburgh Institute \\ Sichuan University, Chengdu, China \\ yuanshengyu1999@outlook.com; gengshuohu1023@gmail.com; Saicheong.fok@scupi.cn
}

\begin{abstract}
Influencing factors are important considerations in the application of non-invasive thermal diagnostics for the early detection of breast tumour. In this paper, experimental studies of artificial tumours embedded inside silicone breasts coupled with numerical simulations using 3D finite-element method in ANSYS were used to investigate the effects of tissue conductivity, tumour size and tumour depth on the heat patterns at the breast skin surface. After validating the numerical breast model, the analysis was extended to examine the heat patterns of a growing tumour. The findings revealed that thermal patterns of the breast surface over time could be useful for the detection of tumours. The existence of tumours would be more noticeable from thermal images of breasts with more fatty tissues, and breasts of lower density. The method would be more suitable for the detection of large tumours near the skin surface. The simulated results suggested that it is possible to detect an initial $4 \mathrm{~mm}$ HER2-positive tumour at a depth of $44 \mathrm{~mm}$ after about 196 days when it had grown to $7 \mathrm{~mm}$ using a temperature sensor with resolution of $0.01{ }^{\circ} \mathrm{C}$.
\end{abstract}

Keywords: thermal images; breast tumour; finite-element method; non-invasive diagnostics

\section{Introduction}

Body temperature is commonly used as a health indicator and thermal data had been applied for the diagnosis of many illnesses [1]. Recently, thermal patterns had been suggested for the early detection of breast tumours [2-4]. This technique is relatively inexpensive, non-invasive, and temperature sensors can be easily embedded into wearable computers for continuous real-time monitoring of health status. These advantages would facilitate the early detection of breast tumours so that the disease can be treated in the early stages, which would ensure higher probability of successful treatment.

The changing thermal characteristics associated with a growing tumour is the result of the rapid volumetric development and acceleration of local metabolism directly related to the speed of cell division [5]. Clinical results had shown that the blood flow to a growing tumour was greater than the blood flow of surrounding normal tissues [6], and the subsurface of the breast tumour lesion is hotter than the surrounding tissues. Gautherie [7] found that the temperature of a cancerous tumour inside the breast can be about $3{ }^{\circ} \mathrm{C}$ higher than normal tissue. As the tumour grows, the heat pattern would change. The escalation in temperature over time on the breast surface for a growing tumour can be captured using temperature sensors or infrared cameras. By comparing the changing temperature profiles between the lift and right breasts with respect to time, abnormality that warrants further investigation can be detected.

Although the concept of using temperature profiles over time for the detection of growing tumour is relatively simple, factors influencing the thermal patterns had not been fully investigated. This paper examines the effects of the changes in tissue conductivity, tumour size and tumour depth on the surface heat patterns. Experimental studies were conducted on breasts made from three types of silicone rubbers with different material properties. Heated resistors were inserted into the artificial breasts to simulate heat generated from tumours of specific sizes and locations. The steady state heat patterns of these breasts were captured using an infrared camera. At the same time, numerical models of these breasts were developed using finite-element method (FEM) in ANSYS. After the verification of the numerical models with experimental results, the FEM model was then extended using realistic tissue properties, body temperature, and tumour heat generation characteristics to examine the changes in heat patterns over time for a growing tumour. Through this study, the resolution of the infrared camera or temperature sensor required for detection of a tumour of a certain size and depth can be determined, along with considerations of factors that could influence the heat patterns. 


\section{Experimental Study and Numerical Simulation}

In the experimental studies, artificial breasts were made from three types of silicone rubbers: T00, T05, and T10. The properties of the silicone rubbers are tabulated in Table 1. Fig. 1a shows a breast casted from T00 using the 3D printed mould in Fig. 1b (the origin "O" and the Cartesian $(x, y, z)$ coordinates are shown in Fig. 1b). Three breasts were made entirely from a single type of silicone rubber, like the one shown in Fig. 1a. In addition, multi-layered breasts were also made using different coatings of silicone rubbers with dissimilar thickness, like the examples shown in Fig. 2.

Resistors (of resistance $R=78 \mathrm{k} \Omega$ with applied voltage of $5.14 \mathrm{~V}$ ) were inserted at location with coordinates $(85$ $\mathrm{mm}, 90 \mathrm{~mm}, 32 \mathrm{~mm}$ ) inside all the breasts to simulate $4 \mathrm{~mm}$ diameter tumours with heat generation rate of $9818 \mathrm{~W} / \mathrm{m}^{3}$.

Before the start of each experiment, the room temperature as well as the temperature of the ground where the breast was placed were measured. The voltage was then applied across the resistors and the heat pattern was captured with the Flir T630sc infrared camera at 20 min intervals. The images were captured perpendicular to the z-axis in the x-y plane. The process stopped after the surface temperature had reached steady state. A final thermograph was taken for each of one-layer breasts after 300 minutes and it took 260 minutes for each of multi-layer breasts to confirm the steady state heat pattern. The process was repeated for all the breasts.

Table.1: Silicone rubbers' properties

\begin{tabular}{|c|c|c|c|c|}
\hline Silicone Type & $\begin{array}{c}\text { Density } \\
\left(\mathrm{kg} / \mathrm{m}^{3}\right)\end{array}$ & $\begin{array}{c}\text { Absolute } \\
\text { Viscosity }(\mathrm{Pa} \cdot \mathrm{s})\end{array}$ & $\begin{array}{c}\text { Tensile Strength } \\
(\mathrm{MPa})\end{array}$ & $\begin{array}{c}\text { Conductivity } \\
\text { Coefficient }(\mathrm{W} / \mathrm{m} \cdot \mathrm{K})\end{array}$ \\
\hline Ecoflex0010 (T00) & 1.12 & 15 & 1.379 & 0.4 \\
\hline Ecoflex0050 (T05) & 1.07 & 2 & $1.441-3.503$ & 0.25 \\
\hline Dragon Skin10 (T10) & 1.07 & 3 & $2.9992-4.999$ & 0.35 \\
\hline
\end{tabular}

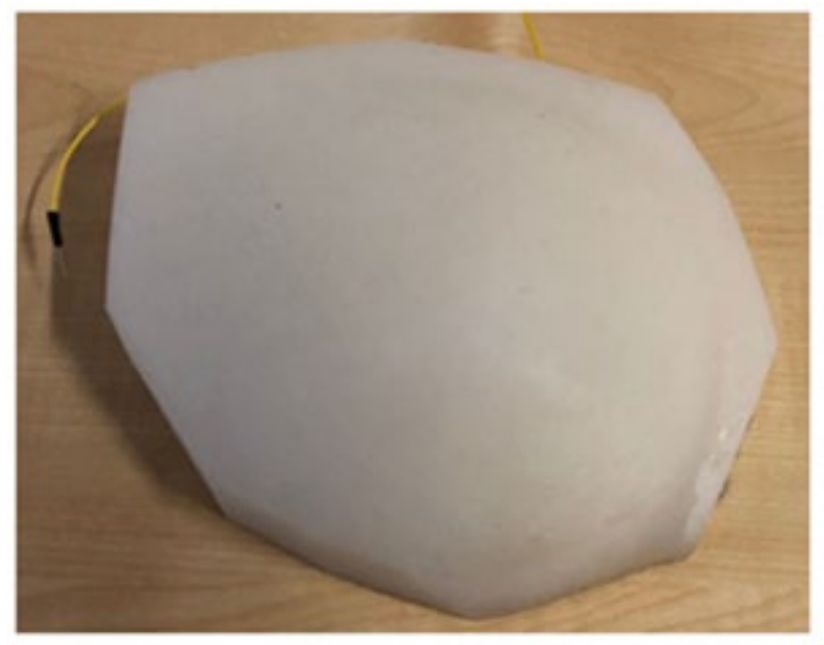

(a) Artificial breast

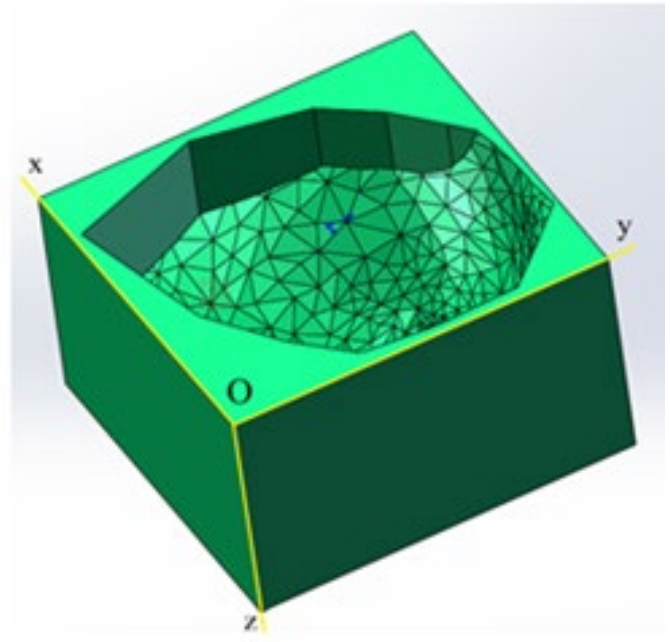

(b) Mould with coordinate axes

Fig. 1: A casted breast with mould

In the numerical model development, the 3D geometrical model of the breast, the heat conduction process along with appropriate equation boundary conditions, were utilized for the finite element analysis. The finite element analysis (FEA) was based on solving Penne's' equation:

$$
\rho c \frac{d T}{d t}=\nabla \cdot(k \nabla T)+\rho_{b} w_{b} c_{b}\left(T_{a}-T\right)+q_{m}
$$


where $k$ is the thermal conductivity of the tissue, $\rho_{b}$ and $c_{b}$ are the density and the specific heat of the blood respectively, $w_{b}$ is the blood perfusion rate $(\mathrm{ml} / \mathrm{s} / \mathrm{ml}), q_{m}$ is the metabolic heat generation rate $\left(\mathrm{W} / \mathrm{m}^{3}\right), T_{a}$ is the arterial blood temperature, and $T$ is the local temperature of the breast tissue. In the steady state, the equation can be expressed as:

$$
k \nabla^{2} T+\rho_{b} w_{b} c_{b}\left(T_{a}-T\right)+q_{m}=0
$$

In the steady state, the heat convection is equal to the conduction inside the breast:

$$
-k \nabla T=h\left(T_{s}-T_{a m}\right)
$$

where $T_{a}=T, h$ is the convection heat transfer coefficient; $T_{s}$ is the surface temperature of the breast; $T_{a m}$ is the ambient temperature, which is the same as the room temperature. The convective heat transfer coefficient, $h=5 \mathrm{~W} /\left(\mathrm{m}^{2} \cdot{ }^{\circ} \mathrm{C}\right)$, was taken from Chanmugam [8]. The tumour size and location in the numerical models and the experimental studies were similar.

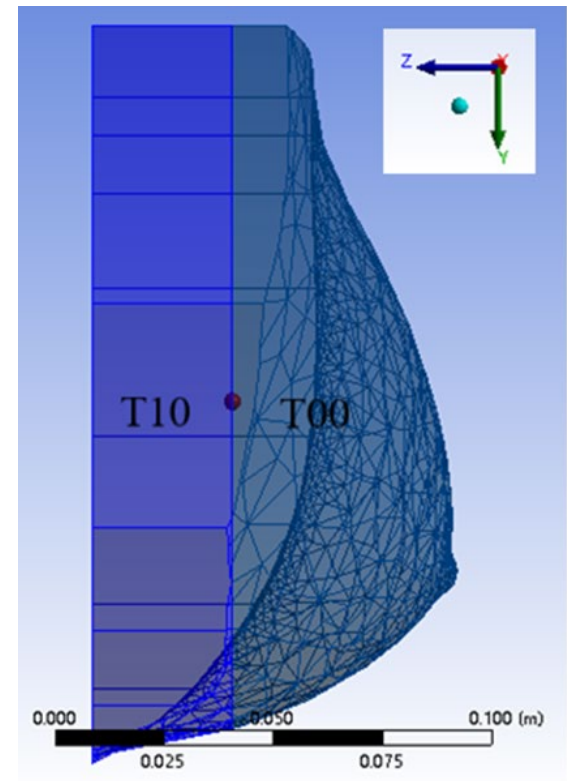

(a) 2-layer breast

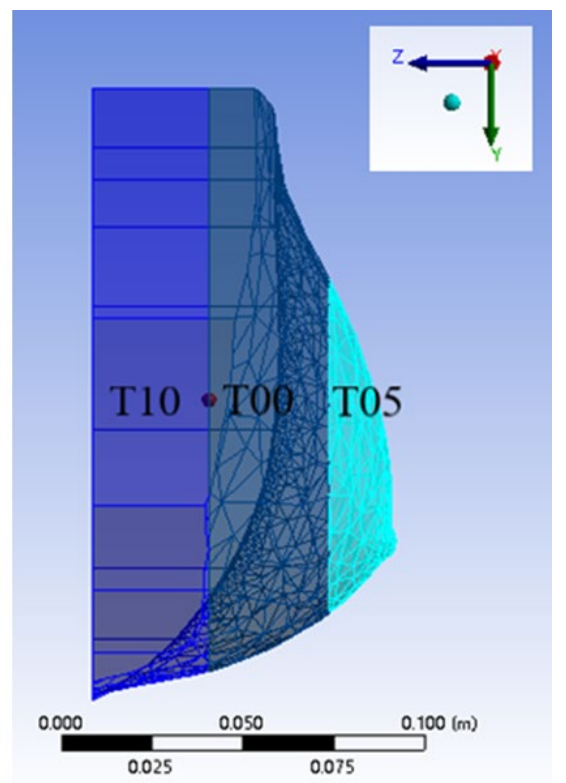

(b) 3-layer breast

Fig.2: Examples of multi-layer breasts

Fig. 3 shows an example of the experimental and simulated temperature profiles for the T05 breasts. The temperature distributions in Fig. 3 show that the experimental and simulated results were very similar. The locations of the maximum and minimum temperatures in the experiments match those in the simulations. All the other experimental and simulated temperature profiles on the breast surfaces were also in good agreement. The errors between the maximum and minimum temperatures for single and multiple-layer breasts were less than $1 \%$. The results verified the numerical model.

Although the experimental and simulated results showed certain trends on the effects of material conductivity on the heat patterns, the comparison was difficult due to the fluctuations of the room and ground temperatures in the experiments. To overcome this problem, the effects of the tissue conductivity, tumour size and tumour depths on the surface temperature profiles were studied using the numerical model under a constant ground temperature of $20^{\circ} \mathrm{C}$ and room temperature of $20.5^{\circ} \mathrm{C}$. 


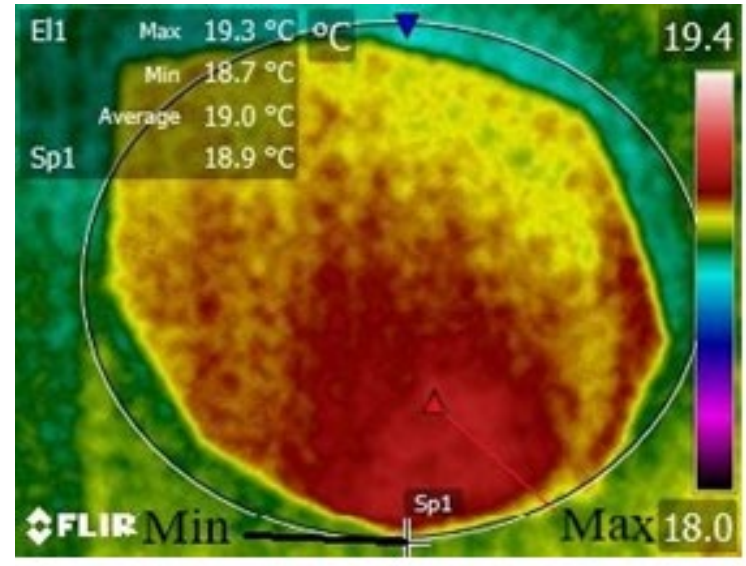

(a) Experiment

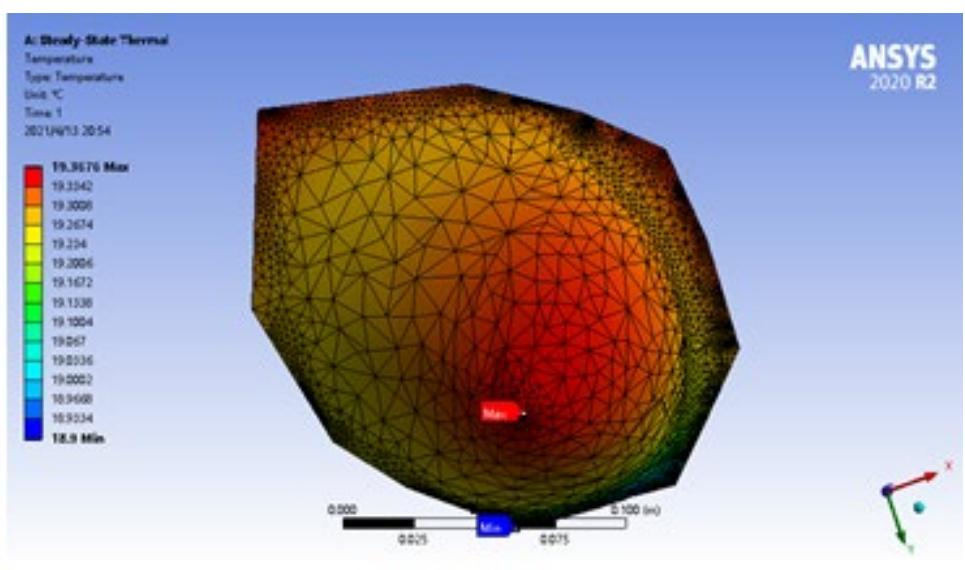

(b) Simulation

Fig. 3: Comparison of experimental and simulated surface temperature profiles for the T05 breast

\section{Effects of Tissue conductivity, Tumour size, and Tumour locations}

\subsection{Tissue Conductivity}

Table 2 tabulates the effect of the material conductivities on the differences between the maximum and minimum temperatures on the breast surfaces for single-layered breasts with a $15 \mathrm{~mm}$ diameter tumour located at Cartesian coordinates $(85 \mathrm{~mm}, 90 \mathrm{~mm}, 32 \mathrm{~mm})$. The temperature difference increases with decreasing conductivity coefficients. The results from the multi-layered breasts also revealed that the layering of tissues with different conductivities in the breast will also affect the surface heat pattern. For two breasts with the similar bottom layers, the differences between the maximum and minimum temperatures on the breast surfaces will decrease when the conductivity of the top layer material increases. When the top layers of two breasts are similar, the temperature difference between the maximum and minimum temperatures on the breast surfaces will increase when the conductivity of the bottom layer material decreases. In some cases, the changes in temperature differences can be too small to be picked up by the sensors with insufficient resolution. The temperature difference in multilayer breasts is dependent on the material combination and is always between that of the materials with the least and largest conductivities.

Table 2: Effect of conductivity on surface temperatures for single-layered breasts

\begin{tabular}{|c|c|c|c|c|c|c|}
\hline $\begin{array}{c}\text { Room } \\
\begin{array}{c}\text { Temperature } \\
\left({ }^{\circ} \mathrm{C}\right)\end{array}\end{array}$ & $\begin{array}{c}\text { Ground } \\
\text { Temperature } \\
\left({ }^{\circ} \mathrm{C}\right)\end{array}$ & $\begin{array}{c}\text { Silicone } \\
\text { Type }\end{array}$ & $\begin{array}{c}\text { Conductivity } \\
\text { Coefficient } \\
(W / m \cdot K)\end{array}$ & $\begin{array}{c}\text { Maximum } \\
\text { Temperature } \\
\left({ }^{\circ} \mathrm{C}\right)\end{array}$ & $\begin{array}{c}\text { Minimum } \\
\text { Temperature } \\
\left({ }^{\circ} \mathrm{C}\right)\end{array}$ & $\begin{array}{c}\text { Temperature } \\
\text { Difference } \\
\left({ }^{\circ} \mathrm{C}\right)\end{array}$ \\
\hline \multirow{2}{*}{20.5} & \multirow{2}{*}{20} & $\mathrm{~T} 00$ & 0.4 & 20.80964 & 20 & 0.80964 \\
\cline { 2 - 7 } & $\mathrm{T} 10$ & 0.35 & 20.86983 & 20 & 0.86983 \\
\cline { 2 - 7 } & $\mathrm{T} 05$ & 0.25 & 21.02505 & 20 & 1.02505 \\
\hline
\end{tabular}

The findings have implications in practical situations. Generally, higher thermal conductivity is associated with higher breast tissue density, which can change with age. The breasts of older women typically have lower density [9]. The result indicates that age can be a consideration factor when using thermal patterns for the detection of breast tumours. The finding suggests that it would be easier to use heat patterns to detect tumours in elderly women. Furthermore, the conductivity of fat is much lower than other tissues and it should be easier to use the approach to detect tumours in breasts with higher fat content. The result is in agreements with findings documented in Omranipour et al. [10]. 


\subsection{Tumour Size}

Fig. 4 shows a typical simulated heat patterns for a T05 breast subjected to a ground temperature of $20^{\circ} \mathrm{C}$ and room temperature of $20.5^{\circ} \mathrm{C}$ with tumour sizes of 12 and $15 \mathrm{~mm}$ at Cartesian coordinates $(85 \mathrm{~mm}, 90 \mathrm{~mm}, 32 \mathrm{~mm})$. The tumour size affects the temperature distribution on the breast surface. It was found that larger tumour will increase the difference between the maximum temperature and the minimum temperature on the breast surface. The maximum surface temperature can be below or above room temperature depending on the tumour size. In general, the hottest point on the surface can shift as the tumour size increases to a location that is directly above the tumour. The shift of the hottest point is due to the breast geometry. The finding indicated that it will be easier to detect larger tumour with thermal images. This finding agrees with the result in [11].

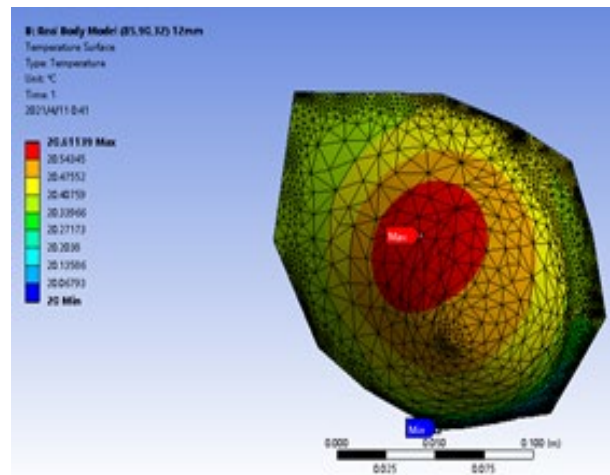

(a) $12 \mathrm{~mm}$ tumour

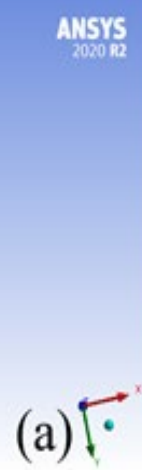

(a)

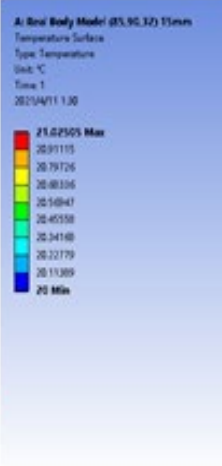

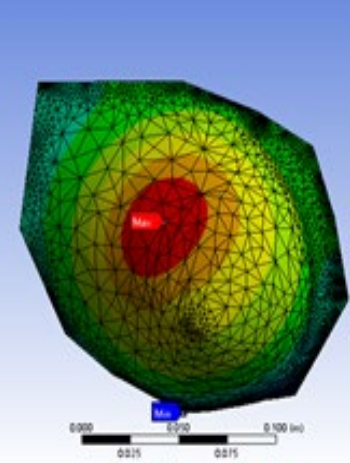

(b) $15 \mathrm{~mm}$ tumour
ANSYS ${ }_{2020}{ }^{2}$

Fig. 4: Surface temperature profiles for T05 breasts with tumour

\subsection{Tumour Depth}

It is well known that it is very difficult to use thermography to detect a small tumour developing deep within the breast. To study this situation, the numerical simulated surface heat patterns of a small $8 \mathrm{~mm}$ tumour at different depths were examined at ground temperature of $20^{\circ} \mathrm{C}$ and room temperature of $20.5{ }^{\circ} \mathrm{C}$. The results for the T05 silicone breast are summarized in Table 3, which shows the maximum and minimum surface temperatures along with their differences. The results indicated that as the tumour approaches the surface, the maximum surface temperature increases, while the minimum surface temperature remains constant at $20^{\circ} \mathrm{C}$. The changes in the tumour depths did not affect the location of the coldest point, which was always located at the bottom corner on the breast surface. However, the location of the hottest point can shift as the tumour approaches the surface. This is mainly due to the geometry of the breast. The effect of the changing locations of the hottest point to a position directly above the tumour is very similar to that encountered in the changing tumour size.

Table 3: Some surface temperatures for an $8 \mathrm{~mm}$ tumour in the T05 breast

\begin{tabular}{|c|c|c|c|}
\hline $\begin{array}{c}\text { Tumour Location } \\
(\mathrm{mm})\end{array}$ & $\begin{array}{c}\text { Maximum Temperature } \\
\left({ }^{\circ} \mathrm{C}\right)\end{array}$ & $\begin{array}{c}\text { Minimum Temperature } \\
\left({ }^{\circ} \mathrm{C}\right)\end{array}$ & $\begin{array}{c}\text { Temperature Difference } \\
\left({ }^{\circ} \mathrm{C}\right)\end{array}$ \\
\hline$(85,90,25)$ & 20.40461 & 20 & 0.40461 \\
\hline$(85,90,32)$ & 20.40967 & 20 & 0.40967 \\
\hline$(85,90,40)$ & 20.41843 & 20 & 0.41843 \\
\hline
\end{tabular}

The result indicated that heat generated from a small tumour at different depths may not significantly influence the temperature difference between the hottest and coldest points on the skin surface. The location of the coldest point on the surface where the temperature remains consistent may also be useful as a reference point for the detection of abnormality through comparison of the heat patterns between the left and right breasts. 


\section{Tumour Growth}

The main advantage of the numerical model is that the simulation can be used to further predict the effects of tumour growth in human breasts on the heat patterns. This is accomplished by changing the parameters and conditions based on tissues' properties and human body temperature. In this study, it is assumed that human breast consists entirely of gland, which has a thermal conductivity of $0.48 \mathrm{~W} / \mathrm{m} \cdot \mathrm{K}$. It is worth noting that the most of breast tissues have conductivity of $0.48 \mathrm{~W} / \mathrm{m} \cdot \mathrm{K}$. This would imply that the assumption is valid if the breast has insignificant amount of fat. The thoracic wall was assumed to be maintain at a constant temperature of $37^{\circ} \mathrm{C}$.

It is reasonable to assume that the heat generation from the tumour will increase with the tumour diameter. The relationship between tumour diameter and heat generated is assumed to be linear, i.e., heat generation varying between 14000 $\mathrm{W} / \mathrm{m}^{3}$ and $70000 \mathrm{~W} / \mathrm{m}^{3}$ for tumour diameter between 0.005 to $0.0015 \mathrm{~m}$. The simulation examined the heat patterns as the tumour diameter increased by $1 \mathrm{~mm}$ starting from $4 \mathrm{~mm}$. The tumour is located at Cartesian coordinates $(85 \mathrm{~mm}, 90 \mathrm{~mm}, 32$ $\mathrm{mm}$ ). The specific growth rates (SGRs) determined by ultrasonography in a recent study [12] for triple-negative tumours and HER2-positive tumours were $10.03 \times 10^{-3}$ day $^{-1}$ and $8.59 \times 10^{-3}$ day $^{-1}$ respectively. A referenced healthy breast with no tumour was also simulated. By comparing the heat patterns of the tumour growth with the referenced breast, the feasibility of using thermal patterns for tumour detection can be examined. The process would be useful to determine the resolution of the thermal sensors needed to distinguish the heat patterns between the breasts with and without tumour at different stages of the tumour growth. The study would also be useful to determine the stage of the tumour growth that could be detected by the thermal patterns. Fig. 5 shows heat patterns as the tumour grows from $4 \mathrm{~mm}$ to $14 \mathrm{~mm}$ diameter.

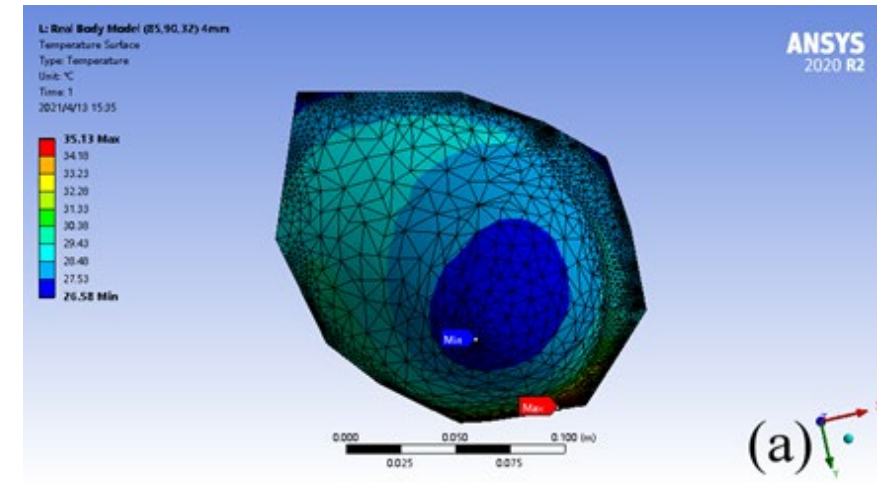

(a) $4 \mathrm{~mm}$ tumour

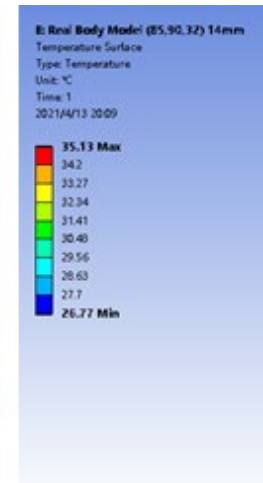

Fig. 5: Tumour growth from $4 \mathrm{~mm}$ to $14 \mathrm{~mm}$

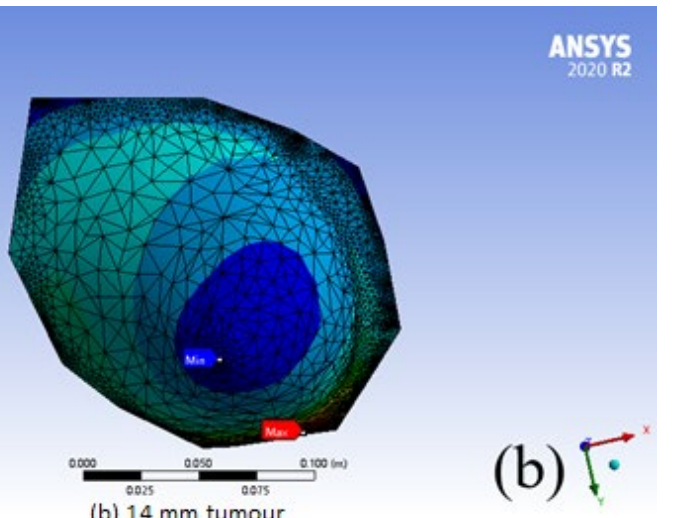

(b) $14 \mathrm{~mm}$ tumour

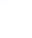

The simulation results of human breast are different from that of the silicone breast. In the experimental studies using silicone breasts, the room temperature was always higher than the ground temperature. However, the temperature of thoracic wall in a human breast is assumed to be constant and always larger than the room temperature. Thus, the hottest point in the human breast is always inside the body while the minimum temperature is on the surface. This is important as the thermogram is $2 \mathrm{D}$ and will only display the surface temperatures. To overcome this issue, the coldest point on the breast surface (discussed in section 3.3) was used as the basis for comparison.

By comparing the differences in surface coldest temperatures between breasts with and without tumours, the resolution of the thermal camera needed and the earliest stage the tumour can be detected could be determined. Based on the numerical simulation results, the thermal camera with a resolution of $0.1{ }^{\circ} \mathrm{C}$ will be able to detect the tumour located at a depth of 44 $\mathrm{mm}$ when the diameter of tumour is $12 \mathrm{~mm}$ (total time of growth from $4 \mathrm{~mm}$ to $12 \mathrm{~mm}$ is about 329 for the triple-negative tumours and 384 days for the HER2-positive tumours). However, using a camera with $0.01{ }^{\circ} \mathrm{C}$ resolution, simulation results indicated that it should be able to detect a $7 \mathrm{~mm}$ tumour at a depth of $44 \mathrm{~mm}$ (total time of growth from $4 \mathrm{~mm}$ to $7 \mathrm{~mm}$ is about 168 days for the triple-negative tumours and 196 days for the HER2-positive tumours). When the diameter is less than this limit, it is hard for the camera to detect the presence of the breast tumour at this depth. 


\section{Conclusion}

This study presents the experimental and numerical investigation on the effects of the changes in tissue conductivity, tumour size, and tumour depth on the heat patterns at the breast surface. Artificial breasts were made using single and multilayered coatings of three silicone rubbers with different material properties. Heated resistors were inserted into these artificial breasts to simulate heat generated from tumours of specific sizes and locations. The heat patterns were captured with an infrared camera. At the same time, numerical models of these breasts were developed using FEM in ANSYS and validated by comparison of the surface heat patterns with the corresponding experimental results. It was determined that the surface heat patterns of the experiments matched the simulated results of similar conditions in terms of temperature distributions along with the magnitudes and locations of the maximum and minimum temperatures. The findings of these silicone breasts revealed that decreasing the thermal conductivity, increasing the tumour size, and decreasing the tumour depth can increase the difference between the maximum temperature and the minimum temperature on the breast surface. For the same breast geometry, the maximum temperature at the breast surface will depend on the thermal conductivity and tumour size as well as tumour depth. These results have direct implications as fatty tissues have lower conductivity and tissue conductivity is also associated with breast density, which generally decreases with age. In addition, the findings indicated that different layers of tissues with different conductivity and thickness can also affect the surface heat patterns.

The result showed that heat generated from a small tumour at different depths may not significantly influence the temperature difference between the hottest and coldest points on the skin surface. For the same breast geometry, increasing the tumour size and decreasing the tumour depth causes the location of the hottest point on the surface to gradually move to a point which is directly above the tumour. The shift in the location of the hottest point is a consequence of the breast geometry and would be useful as the starting point for the inverse mapping to determine the tumour location using 2D thermal patterns. On the other hand, the coldest point is always on the breast surface. The temperature and location of the coldest point did not shift significantly with tumour depth. As a result, the coldest point may be useful as a reference for the detection of abnormality through comparison of the left and right breasts.

Finally, the FEA were extended to examine the changes in heat patterns over time for a growing tumour in a human breast without fatty tissues. By comparing the differences in surface temperatures between breasts with and without tumours, it was found that the thermal camera with a resolution of $0.1{ }^{\circ} \mathrm{C}$ will be able to detect the tumor located at a depth of $44 \mathrm{~mm}$ when the diameter of tumor is $12 \mathrm{~mm}$ (total time of growth from $4 \mathrm{~mm}$ to $12 \mathrm{~mm}$ is about 329 days for the triple-negative tumours and 384days for the HER2-positive tumours). However, using a camera with $0.01{ }^{\circ} \mathrm{C}$ resolution, simulation results indicated that it should be able to detect a $7 \mathrm{~mm}$ at a depth of $44 \mathrm{~mm}$ with the time for detection reduced by half (i.e., total time of growth from $4 \mathrm{~mm}$ to $7 \mathrm{~mm}$ is about 168 days for the triple-negative tumours and 196 days for the HER2-positive tumours). Hence, to detect a growing tumour earlier, the improvement of the sensor resolution is critical.

\section{Acknowledgements}

The authors want to thank Prof. Y. Zhao of Nazarbayev University for sharing the breast geometry used in his work.

\section{References}

[1] D. Zhong-San, and L. Jing. "Mathematical modelling of temperature mapping over skin surface and its implementation in thermal disease diagnostics," Comput Biol Med., vol. 34(6), pp. 495-521, 2004.

[2] S.V. Francis, M. Sasikala, and S.D. Jaipurkar, "Detection of Breast Abnormality Using Rotational Thermography," in Application of Infrared to Biomedical Sciences, Springer, Singapore, pp. 133-158, 2017.

[3] E. Y-K. Ng and S.C. Fok, "A Framework for Early Discovery of Breast Tumour using Thermography with Artificial Neural Network", The Breast Journal, vol. 9, pp. 341- 343, 2003.

[4] O. Mukhmetov, D. Igali, A. Mashekova, Y. Zhao, E.Y.K. Ng, S. Fok and S.L. Teh, "Thermal modeling for breast tumour detection using thermography," International Journal of Thermal Sciences, DOI: 10.1016/j.ijthermalsci.2020. $106712,2020$. 
[5] C. Guiot, P.G. Degiorgis, P.P. Delsanto, P. Gabriele and T.S. Deisboeck, "Does tumour growth follow a universal law," Journal of Theoretical Biology, vol. 225, pp. 147-151, 2003.

[6] A. Talkington and R. Durrett, "Estimating tumour growth rates in vivo," Bull Math Biol, vol. 77, pp. 1934-1954, 2015.

[7] M. Gautherie, "Temperature and blood flow patterns in breast cancer during natural evolution and following radiotherapy," Biomed. Thermology, vol. 107, pp. 21-64, 1982.

[8] A. Chanmugam, R. Hatwar and C. Herman, "Thermal analysis of cancerous breast model," Proc. Int Mech Eng Congress Expo, pp. 134-143, 2012.

[9] C.M. Checka, J.E. Chun, F.R. Schnabel, J. Lee and H. Toth, "The relationship of mammographic density and age: implications for breast cancer screening," America Journal of Roentgenology, vol.198, no.3, pp. 292-295, 2012.

[10] R. Omranipour, A. Kazemian, S. Alipour, M. Najafi, M. Alidoosti, M. Navid, A. Alikhassi, N. Ahmadinejad, K. Bagheri, S. Izadi, "Comparison of the Accuracy of Thermography and Mammography in the Detection of Breast Cancer," Breast Care, vol. 11, pp. 260-264, 2016.

[11] O. Mukhetovf, D. Iglai, Y. Zhao, S.C. Fok, S. Lee and A. Mashekova, "Finite element modelling for the detection of breast tumour," in Proc. Of the IEEE 18th International Conference on Bioinformatic and Bioengineering, Taiwan, 2018, pp. 360-363.

[12] S.H. Lee, Y.S. Kim, W. Han, H.S. Ryu, J.M. Chang, N. Cho and W.K. Moon, "Tumor growth rate of invasive breast cancers during wait times for surgery assessed by ultrasonography," Medicine, vol. 95(37), 2016. 